\title{
El ABCD en prevención del paludismo en niños viajeros
}

The $A B C D$ of malaria prevention in pediatric travelers

\author{
Dra. Griselda Berberian ${ }^{a}$, Dra. M. Teresa Rosanova ${ }^{b}$, Dra. Cecilia Torroija ${ }^{c}$ y \\ Dra. M. Laura Praino ${ }^{d}$
}

\section{RESUMEN}

Los movimientos poblacionales y de viajeros a áreas endémicas de paludismo, su diseminación y la aparición de cepas resistentes han llevado al resurgimiento de esta enfermedad como problema global de la salud. Se estiman 660000 muertes por año por el paludismo, cuya población más vulnerable son los niños menores de 5 años y las embarazadas. El conocimiento de la enfermedad y las medidas generales para la prevención de las picaduras de insectos son las medidas básicas y fundamentales para la prevención. La utilización de quimioprofilaxis antipalúdica complementa el resto delas medidas y debe ser evaluada sobre la base del riesgobeneficio de cada situación en particular.

Palabras clave: paludismo, profilaxis, niños.

\section{ABSTRACT}

The development and spread of drug resistant Malaria parasites, population and travelers movements to Malaria zones have led to the resurgence of Malaria as a global health problem. Estimates suggest that 660000 deaths occur annually, mainly in infants, children and pregnant woman. Disease knowledge and protection against mosquito bites are the first line of defense against Malaria. Malaria chemoprophylaxis adds to these measures, it must be evaluated based on the individual risk. Key words: malaria, prophylaxis, children.

http:/ /dx.doi.org/10.5546/aap.2014.468

\section{INTRODUCCIÓN}

El paludismo es la parasitosis endémica de mayor prevalencia mundial distribuida en áreas tropicales y subtropicales de más de 100 países de África, Asia (Sur, Sudeste y Medio Oriente), Centroamérica, Caribe y la Amazonia en Sudamérica (Figura 1). La región del África concentra más del $80 \%$ de los casos y el $90 \%$ de las muertes por la enfermedad. La presencia de la enfermedad, a su vez, tiene una amplia variabilidad estacional durante el año y está en relación directa con las temporadas de lluvias.
El 40\% de la población mundial más pobre vive en áreas de riesgo de paludismo. Para el año 2010, la OMS estimó 500 millones de casos de paludismo en el mundo, con una reducción a 219 millones para $2012{ }^{1}$

De acuerdo con los datos del informe mundial sobre paludismo del año 2012 de la OMS, los sistemas de vigilancia indican que 50 países están en vías de reducir la incidencia de casos en un $75 \%$ para el 2015 ; la mayor parte de ellos corresponden a la región de las Américas, que representa solo el 3\% de la prevalencia mundial (Figura 2).

En Argentina, el programa nacional tiene por objeto una vigilancia sistematizada para la detección temprana de casos de paludismo, así como la planificación de acciones tendientes a eliminar el mosquito que transmite la enfermedad. Antes de su implementación, en Argentina, se notificaban entre 100 y 200 personas con paludismo por año, todos casos circunscriptos al norte del país, de los cuales entre el 50 y el $70 \%$ eran importados de Bolivia y Paraguay. ${ }^{2}$

A partir de 2010, considerando la estrategia nacional contra el paludismo, se pronunció una tendencia decreciente de casos. Argentina se encuentra en la fase de preeliminación de la enfermedad ya que no presenta casos autóctonos desde el año 2009.

El paludismo no es solo un problema de los países en vías de desarrollo, ya que con el incremento de los viajes internacionales en los últimos años existe un aumento en el número de viajeros en riesgo de contraer la enfermedad. Se estima 

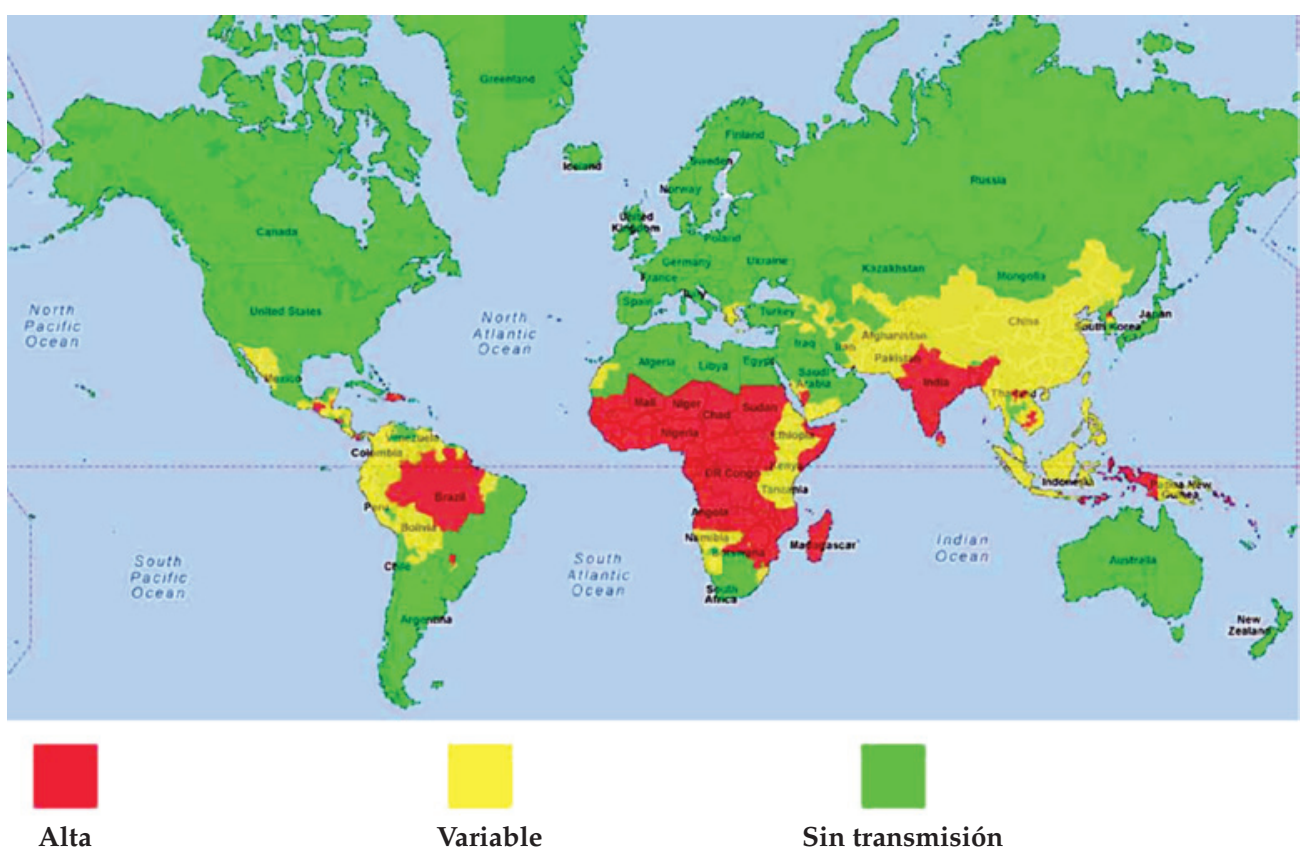

\section{Sin transmisión}

Fuente: Center of Global Health. Division of Parasitic Diseases and Malaria.

www.cdc.gov/malaria/travelers/country_table/a.html

Figura 2. Tendencia de casos de paludismo 2000-2011

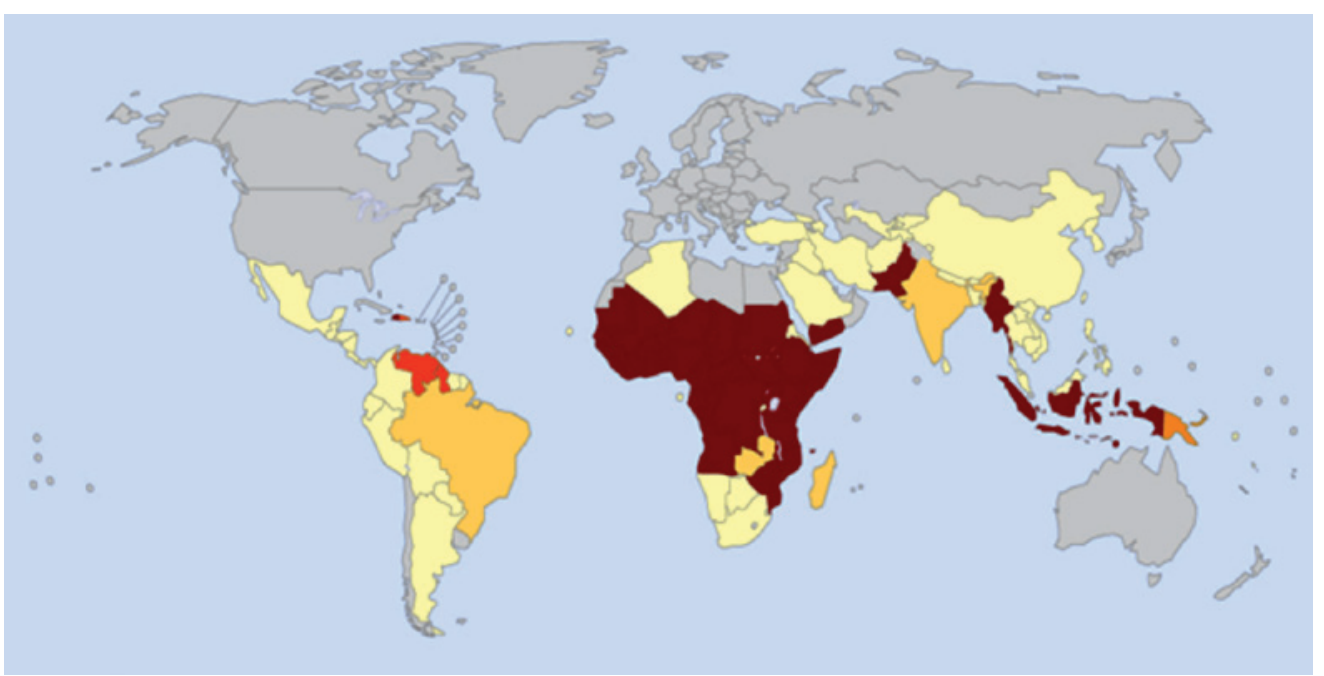

No aplicable o libre de paludismo.

En seguimiento por disminución del 75\% de incidencia durante 2000-2015.

50-75\% de disminución en la incidencia proyectada durante 2000-2015.

$<50 \%$ de disminución en la incidencia proyectada durante 2000-2015.

Aumento en la incidencia durante 2000-2011.

Datos inconsistentes.

Fuente: OMS, Reporte Mundial de paludismo, 2012. 
que alrededor de 80-90 millones de viajeros visitan áreas endémicas de paludismo y 30000 de ellos adquieren la enfermedad cada año. El $10 \%$ corresponde a niños menores de 17 años. En el caso de paludismo por $P$. falciparum, el 85\% lo adquieren en el continente africano. El riesgo de enfermar difiere entre los viajeros y en las distintas regiones aun en el mismo país. En el año 2010, se reportaron al CDC 1700 casos de malaria, el mayor número de denuncias desde 1980. De estos, el 65\% la habían adquirido en África, 19\% en Asia, $15 \%$ en Caribe y la región de las Américas y menos del 1\% en Oceanía. Teniendo en cuenta el número de viajeros por región el mayor riesgo fue para aquellos que viajaron a África occidental y Oceanía. ${ }^{3}$

Dentro de los viajeros internacionales en edad pediátrica, entre los que se encuentran 1591 niños que volvieron a los Estados Unidos desde 218 destinos durante un período de 10 años (1997-2007), de acuerdo con los datos reportados por el sistema de vigilancia de enfermedades en viajeros (GeoSentinel Survillance Network), que incluyen las patologías del posviaje, el paludismo fue el diagnóstico principal de la fiebre sin foco con el 35\% de los casos, seguido por las infecciones virales (28\%). El viaje de más de 30 días con destino al continente africano y la visita a familiares y amigos fueron los factores de riesgo más frecuentemente relacionados con la adquisición.

El agente etiológico es el protozoario del género Plasmodium, del que existen 4 especies distintas, que son el $P$. vivax, $P$. falciparum, $P$. malariae y $P$. ovale. Los más frecuentes son el paludismo por $P$. falciparum y por $P$. vivax, y el más mortal, el paludismo por P. falciparum.

En los últimos años, también ha habido algunos casos humanos por P. knowlesi, un parásito del mono que aparece en zonas boscosas de Asia Sudoriental.

Todas las especies se transmiten por la picadura de la hembra infectada del mosquito del género Anopheles. Raramente, puede transmitirse por transfusiones, trasplante de órganos, a través de agujas y por transmisión congénita de la madre infectada al feto. ${ }^{4}$

En el mundo, hay unas 20 especies diferentes de Anopheles que tienen importancia local. Todas las especies importantes como vector pican por la noche. Estos mosquitos se crían en agua dulce de poca profundidad (charcos, campos de arroz o huellas de animales).

Es una enfermedad potencialmente grave y letal dependiendo del agente infectante, la inmunidad previa y el tipo de huésped. Se estiman 660000 muertes por años en relación con la enfermedad.

Diagnóstico: La fiebre es el síntoma clave para el diagnóstico y puede estar acompañada de cefalea, sudoración, escalofríos, malestar general, vómitos, diarrea o por un cuadro pseudogripal días, meses o años después del viaje a zonas endémicas, dependiendo de la especie involucrada. El 95\% de las manifestaciones clínicas ocurren dentro del mes para P. falciparum ${ }^{5}$ y pueden ser más tardías y llegar hasta el año posterior al viaje a áreas endémicas para las otras especies, como el $P$. vivax. Las infecciones por este último son raramente fatales.

En el hombre, los parásitos se multiplican en el hígado y luego infectan los glóbulos rojos, razón por la cual la presencia de anemia es característica en el diagnóstico de la enfermedad.

El diagnóstico de paludismo se basa en el examen microscópico de la sangre por técnicas de gota gruesa y frotis fino, que busca las formas típicas de los eritrocitos parasitados sumados a los criterios clínicos y epidemiológicos.

Complicaciones: Los niños tienen menor riesgo respecto de los adultos de desarrollar insuficiencia renal aguda, edema de pulmón e ictericia, pero son más propensos a presentar convulsiones, acidosis láctica, hipoglucemia y anemia grave. ${ }^{2}$

La mortalidad por paludismo por $P$. falciparum varía del 0,6 al 3,8\% y puede llegar a más del $20 \%$ en los casos de paludismo grave, aun en las mejores condiciones de manejo hospitalario. ${ }^{6}$

Las medidas de prevención están destinadas a evitar las picaduras de insectos, al conocimiento de la enfermedad para un rápido diagnóstico y tratamiento, y, por último, a la profilaxis antiparasitaria (quimioprofilaxis). Resulta importante destacar también que, dentro de las actividades preventivas, se incluyen las actividades de control en terreno que nunca deben faltar, como la utilización de insecticidas en el manejo de los casos sintomáticos o en casos de brote.

La necesidad de quimioprofilaxis debe ser evaluada en cada situación en particular dependiendo del riesgo de enfermar en función del destino, tiempo, tipo de viaje y tipo de huésped. ${ }^{7}$

La indicación de la quimioprofilaxis es solo una de las medidas de prevención, ya que ninguna es $100 \%$ eficaz por sí sola, por lo que 
resulta primordial complementarla con el resto de las medidas preventivas.

Son 4 los puntos fundamentales relacionados con la prevención del paludismo en el viajero:

1) Conocer los riesgos del paludismo, el período de incubación, los síntomas principales y la potencial gravedad del cuadro. En cuanto al riesgo, es importante determinar el área geográfica, el estilo de viaje, el tiempo de estadía, la época del año, la altura y las características individuales del viajero.

2) Evitar las picaduras de insectos con medidas generales, como el uso de ropa de mangas y pantalones largos, evitar el uso de perfumes, colocarse repelentes durante todo el día, en especial al anochecer. Usar mosquiteros durante la noche. Las ropas rociadas con permetrina son útiles, así como el uso de insecticidas. Respecto de los repelentes, los más útiles son los que contienen $N$, $N$-dimethyl-m-toluamida (DEET), que pueden utilizarse a partir de los 2 meses de edad, y la picaridina para los mayores de 2 años (esta última no está disponible en nuestro medio).

3) Utilizar quimioprofilaxis con drogas antiparasitarias para evitar la infección en situaciones de alto riesgo.

4) Consultar inmediatamente en caso de fiebre durante y en el posviaje.

\section{Quimioprofilaxis}

La prevención de la malaria para personas no inmunes que visitan zonas de paludismo endémico es complicada debido a la falta de datos exactos sobre el riesgo de infección en viajeros, discordancia entre guías de estrategias de manejo de distintas instituciones, disponibilidad de medicamentos, costo y el riesgo de los efectos adversos relacionados con las drogas. ${ }^{8}$

Por ello, para la indicación de la profilaxis antiparasitaria, es necesario considerar lo siguiente:
1. El riesgo de paludismo de acuerdo con los datos epidemiológicos locales. Algunos autores sugieren no indicarlo para aquellos que viajen a zonas con riesgo de paludismo $<10$ casos/1000 habitantes.

2. La decisión de la profilaxis debe evaluarse sobre la base del riesgo de enfermar o de presentar un efecto adverso por la medicación. En todos los casos, debe evaluarse el riesgobeneficio de la medicación.

3. Utilizar la droga disponible con menos efectos adversos sobre la base de la resistencia del lugar (Tabla 1).

Luego de la decisión de la necesidad o no de la quimioprofilaxis antiparasitaria, la elección de la droga depende fundamentalmente del destino del viaje, y se considera el parásito circulante y los niveles de resistencia local, la presencia o no de patología de base y la edad del niño. ${ }^{9}$

La resistencia parasitaria varía de acuerdo con el área geográfica considerada, por lo que resulta primordial su conocimiento al momento de elegir la profilaxis adecuada del viajero que visita las áreas endémicas.

Las drogas disponibles para los niños son las mismas que para la población adulta, y se tiene en cuenta para cada una la edad y peso del paciente. ${ }^{10}$

Las drogas con probada utilidad para la profilaxis del paludismo son 6: cloroquina, mefloquina, atovaquone/proguanil, doxiciclina (> 8 años), primaquina y proguanil.

Cada una de ellas tiene características propias de eficacia, tolerancia y costo; las más utilizadas son el atovaquone/proguanil, doxiciclina y la mefloquina.

En el cuadro adjunto, se indican las opciones de drogas para la profilaxis, dosis y efectos adversos para considerar (Tabla 2).

En la Tabla 3, se resume en forma práctica la administración por peso de la cloroquina, mefloquina, doxiciclina y atovaquone/proguanil.

TABLA 1. Opciones de profilaxis antipalúdica según el área de resistencia

\begin{tabular}{lcc}
\hline Lugar & Droga/s de elección & Alternativas \\
\hline Sin resistencia a cloroquina & Cloroquina & $\begin{array}{c}\text { Doxiciclina o atovaquone/proguanil o } \\
\text { cloroquina + proguanil }\end{array}$ \\
Con resistencia a cloroquina & Mefloquina o atovaquone/ & Primaquina o \\
proguanil o doxiciclina & cloroquina + proguanil \\
con resistencia a & Doxiciclina o atovaquone/ & \\
\hline
\end{tabular}


En cada situación, deben evaluarse también las ventajas y desventajas de cada una de las drogas por utilizar (Tabla 4) y las interacciones con otras drogas y/o inmunizaciones.
Todos los esquemas de quimioprofilaxis requieren, para ser efectivas, de la toma de la medicación antes, durante y después del viaje al área endémica. Entre ellas, difieren de

TABLA 2. Drogas para el uso de profilaxis antipalúdica en niños

\begin{tabular}{|c|c|c|c|}
\hline & Indicación & Dosis pediátrica & Dosis para adultos \\
\hline \multicolumn{4}{|l|}{ Fosfato de cloroquina (Aralen ${ }^{\circledR}$ ) } \\
\hline Comp.: $150 \mathrm{mg}$ base/300 mg base & Cualquier edad & $5 \mathrm{mg} / \mathrm{kg}$ base semanal & $300 \mathrm{mg}$ \\
\hline $\begin{array}{l}\text { Mefloquina }\left(\text { Lariam }^{\circledR} \text {, } \text { Tropicur }^{\circledR} \text { ) }\right. \\
\text { Comp.: } 250 \mathrm{mg}\end{array}$ & $>5 \mathrm{~kg}$ & $\begin{array}{l}5 \mathrm{mg} / \mathrm{kg} \\
\text { semanales }\end{array}$ & $250 \mathrm{mg}$ \\
\hline $\begin{array}{l}\text { Atovaquone/proguanil (Malarone }{ }^{\circledR} \text { ) } \\
\text { Comp.: } 250 / 100 \mathrm{mg}\end{array}$ & $\begin{array}{l}>5 \mathrm{~kg}(\mathrm{CDC}) \\
>11 \mathrm{~kg}(\mathrm{OMS})\end{array}$ & $\begin{array}{c}4 / 1,6 \mathrm{mg} / \mathrm{kg} / \text { día } \\
\text { diarios }\end{array}$ & $250 / 100 \mathrm{mg}$ \\
\hline $\begin{array}{l}\text { Doxiciclina }\left(\text { Vibramicina }{ }^{\circledR}\right) \\
\text { Comp.: } 100 \mathrm{mg}\end{array}$ & $>8$ años & $\begin{array}{l}1,5-2 \mathrm{mg} / \mathrm{kg} / \text { día } \\
\text { diarios }\end{array}$ & $100 \mathrm{mg}$ \\
\hline $\begin{array}{l}\text { Primaquina } \\
\text { Comp.: } 15 \mathrm{mg} \text { base }\end{array}$ & $\begin{array}{l}>4 \text { años (OMS) } \\
\text { Cualquier edad (CDC) }\end{array}$ & $\begin{array}{l}\text { 0,5 mg base / kg/día } \\
\text { diarios (con alimento) }\end{array}$ & $30 \mathrm{mg}$ \\
\hline $\begin{array}{l}\text { Proguanil (Paludrine }{ }^{\circledR} \text { ) } \\
\text { Comp.: } 100 \mathrm{mg}\end{array}$ & Cualquier edad & $3,5 \mathrm{mg} / \mathrm{kg}$ diarios & $\begin{array}{l}\text { Solo combinado con } \\
\text { cloroquina } 200 \mathrm{mg}\end{array}$ \\
\hline
\end{tabular}

TABLA 3. Administración práctica de referencia por peso/edad de profilaxis antipalúdica

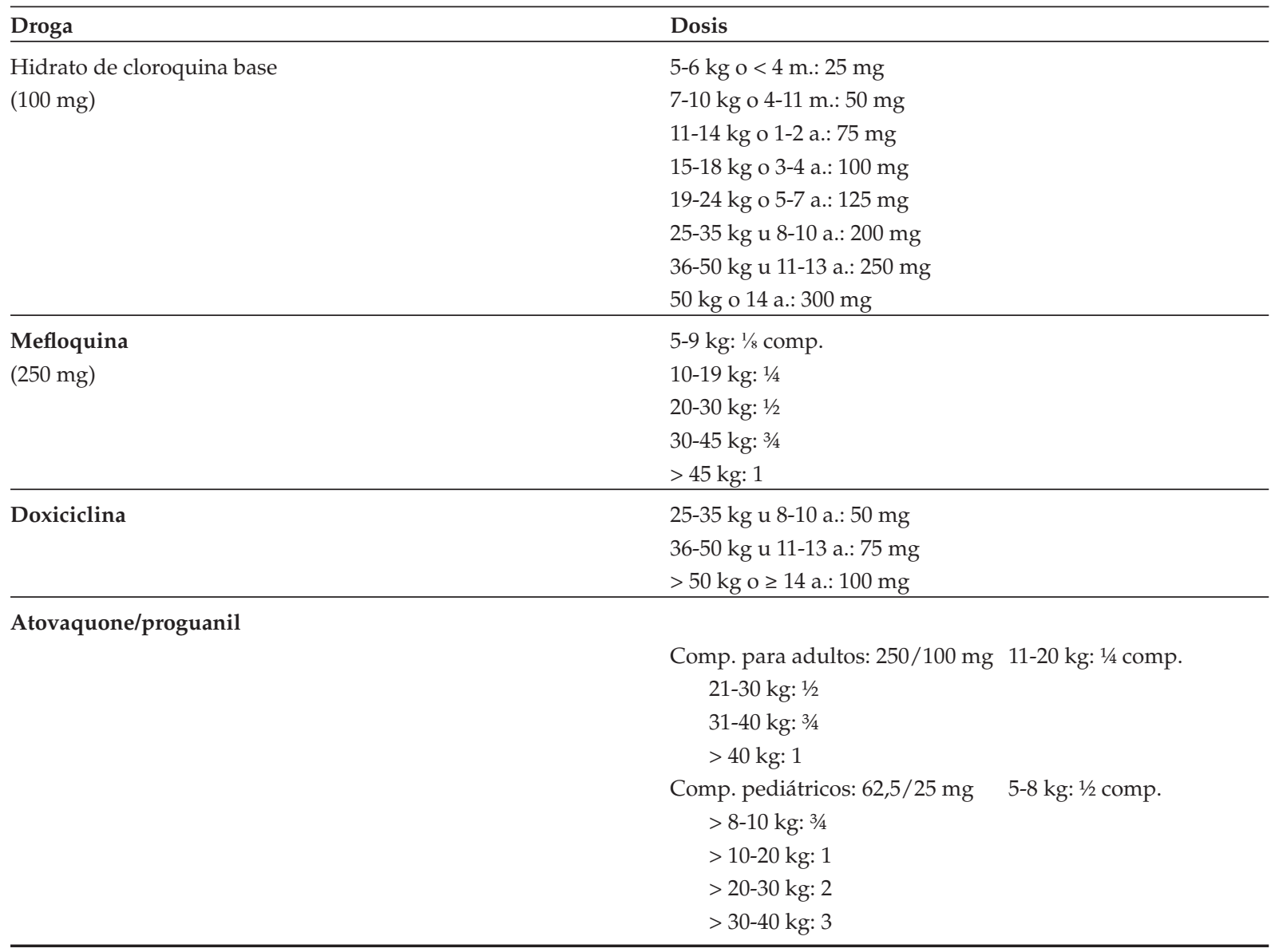


TABla 4. Ventajas y desventajas de las drogas para la profilaxis antipalúdica

\begin{tabular}{|c|c|c|}
\hline & Ventajas & Desventajas \\
\hline $\begin{array}{l}\text { Hidrato de cloroquina } \\
\left(\text { Aralen }^{\circledR}\right)\end{array}$ & De elección para viajes largos & $\begin{array}{l}\text { Gastrointestinales. Visión borrosa. } \\
\text { Exantema. Uso limitado por resistencia }\end{array}$ \\
\hline $\begin{array}{l}\text { Mefloquina } \\
\left(\text { Lariam }^{\circledR}, \text { Tropicur }^{\circledR}\right)\end{array}$ & $\begin{array}{l}\text { De elección para viajes largos. } \\
\text { Puede indicarse en el embarazo }\end{array}$ & $\begin{array}{l}\text { Gastrointestinales. Insomnio. } \\
\text { Sabor amargo. Contraindicada en pacientes } \\
\text { con enfermedades psiquiátricas, } \\
\text { convulsiones, arritmias }\end{array}$ \\
\hline $\begin{array}{l}\text { Atovaquone/proguanil } \\
\left.\text { (Malarone }^{\circledR}\right)\end{array}$ & $\begin{array}{l}\text { De elección para viajes cortos y } \\
\text { Qp. de último momento. Buena tolerancia oral. } \\
\text { Hay comprimidos pediátricos }\end{array}$ & $\begin{array}{l}\text { Gastrointestinales. Aumento de GOT/GPT. } \\
\text { Alto costo. Contraindicada en } \\
\text { embarazadas y lactancia }\end{array}$ \\
\hline $\begin{array}{l}\text { Doxiciclina } \\
\left(\text { Vibramicina }^{\circledR}\right)\end{array}$ & $\begin{array}{l}\text { De elección para viajes cortos y de } \\
\text { último momento. Previene también de otras infecciones, } \\
\text { como ricketsiosis, leptospirosis, etc. }\end{array}$ & $\begin{array}{l}\text { Gastrointestinales. Fotosensibilidad. } \\
\text { Contraindicado en menores de } 8 \text { años }\end{array}$ \\
\hline Primaquina & De elección para viajes cortos y de último momento & $\begin{array}{l}\text { Contraindicada en pacientes con déficit } \\
\text { de G6PD, embarazadas y lactancia. } \\
\text { Requiere zonas con }>90 \% \text { de } P . \text { vivax }\end{array}$ \\
\hline $\begin{array}{l}\text { Proguanil } \\
\left.\text { (Paludrine }^{\circledR}\right)\end{array}$ & $\begin{array}{c}\text { No se utiliza, salvo que no exista } \\
\text { otra opción terapéutica }\end{array}$ & Solo combinado \\
\hline
\end{tabular}

Qp.: quimioprofilaxis.

acuerdo con las características farmacocinéticas y requieren una posología y frecuencia de administraciones diferentes, que las hacen más o menos atractivas para el cumplimiento.

Cloroquina y mefloquina: 1 vez por semana, comenzando 1-3 semanas antes, durante todo el viaje y 4 semanas después de la salida del área endémica.

Doxiciclina y proguanil: 1 vez por día, comenzando 1-2 días antes del viaje, durante este y 4 semanas después de la salida del área endémica.

Atovaquone/proguanil y primaquina: 1 vez por día, comenzando 1-2 días antes del viaje, durante este y solo una semana posviaje por ser activos en la fase hepática de la enfermedad.

\section{CONCLUSIÓN}

El pilar para el manejo de la prevención de la malaria es el conocimiento de la enfermedad y las medidas generales de prevención para las picaduras de insectos.

La utilización de la quimioprofilaxis complementa dichas medidas y está justificada para aquellas personas que viajen a áreas de alto riesgo, por lo que es necesario evaluar el riesgo vs. el beneficio de su utilización en cada situación particular.

Por último, se debe recordar que la fiebre en un viajero que estuvo en área de riesgo de paludismo puede ser el único marcador de enfermedad, por lo que siempre debe ser considerado el paludismo dentro de los diagnósticos diferenciales.

\section{REFERENCIAS}

1. World Health Organization. World Malaria Report 2012. Geneva, 2014. Disponible en: http://www.who. int/malaria/publications/world_malaria_report_2012/ report/en/. [Consulta: 3 de abril de 2014].

2. World Health Organization. World Malaria Report 2013. Geneva, 2014. Disponible en: http://www.who.int/ malaria/publications/world_malaria_report_2013/en/. [Consulta: 21 de abril de 2014].

3. Centers for Disease Control and Prevention. Infectious diseases related to travel. Atlanta, 2013. Disponible en: http: / / wwwnc.cdc.gov/travel/yellowbook/2014/ chapter-3-infectious-diseases-related-to-travel/malaria. [Consulta: 25 de abril de 2014].

4. WhiteNJ.Malaria.En:CookG,Zumla A,eds.Manson'sTropical Diseases. 22 ${ }^{\text {nd }}$ ed. London: Saunders; 2009.Págs.1201-300.

5. Genton B, D'Acremont V. Clinical features of malaria in travelers and migrants. En: Schlagenhauf-Lawlor P, ed. Travelers' malaria. $2^{\text {nd }}$ ed. Hamilton: BC Decker; 2008. Págs.269-83.

6. Askling HH, Nilsson J, Tegnelli A, Janzon R, et al. Malaria risk in travelers. Emerg Infect Dis 2005;11(3):436-41.

7. Schlagenhauf-LawlorP,KainK.MalariaChemoprophylaxis. En: Keystone J, Kozarsky P, Freedman D, Nothdurft H, Connor B, eds. Travel Medicine. $2^{\text {nd }}$ ed. Philadelphia: Elsevier; 2008. Págs.137-57.

8. Schlagenhauf P,PetersenE. Malaria chemoprophylaxis: strategies for risk groups. Clin Microbiol Rev 2008;21(3):466-72.

9. Freedman DO. Malaria prevention in short-term travelers. N Engl J Med 2008;359(6):603-12.

10. Centers for Disease Control and Prevention. Choosing a Drug to Prevent Malaria. Atlanta, 2012. Disponible en: http:/ / www.cdc.gov/malaria/travelers/drugs.html. [Consulta: 3 de abril de 2014]. 